\title{
[EN-A-31]Analysis of Weather Impact on Flight Efficiency for Stockholm Arlanda Airport Arrivals (EIWAC 2019)
}

\author{
${ }^{+}$Anastasia Lemetti ${ }^{1}$, Tatiana Polishchuk ${ }^{1}$, Raúl Sáez ${ }^{2}$, and Xavier Prats ${ }^{2}$ \\ ${ }^{1}$ Communications and Transport Systems, ITN, Linköping University, Norrköping, Sweden \\ ${ }^{2}$ Department of Physics, Technical University of Catalonia (UPC), Castelldefels, Barcelona, Spain
}

\begin{abstract}
Analysis of punctuality of airport arrivals, as well as identification of causes of the delays within transition airspace, is an important step in evaluating performance of the Terminal Maneuvering Area (TMA) Air Navigation Services. In this work we analyse how different weather events influence arrival punctuality and vertical flight efficiency on example of Stockholm Arlanda airport. We quantify the impact of the deviations from the flight plans influenced by different weather events, by demonstrating that they result in significant arrival delays, vertical inefficiencies and calculating how much extra fuel is wasted due to vertical flight inefficiency within Stockholm TMA. ${ }^{1}$
\end{abstract}

Keywords: Vertical Flight Efficiency, Punctuality, Weather Impact.

\section{Introduction}

Aviation, probably more than any other mode of transportation, is greatly affected by weather. Wind direction and speed can make a flight time quite different, for exactly the same journey. Flight routes can be altered to avoid convective weather, such as thunderstorms. Severe weather causes delays and cancellations. Airport capacity can be reduced considerably by low visibility, strong winds, thunderstorms in the terminal area and runway closures.

There are several measures of convective weather. The most common is Convective Available Potential Energy (CAPE), which is an indicator of atmospheric instability that can predict severe weather. When the visibility at the airport drops below Runway Visual Range (RVR), the airport enforces Low Visibility Procedures (LVPs). LVPs mean procedures applied at an aerodrome for the purpose of ensuring safe operations such as spacing planes apart to reduce the chance of collisions, so it slows things down and decreases the airport capacity which in turn leads to delays and cancellations.

Reducing fuel waste has a significant environmental benefit as it reduces fuel emissions. One of the reasons for fuel waste is inefficient vertical profiles. During the descent phase Vertical Flight Efficiency (VFE) means

1. This research is a part of the IFWHEN project supported by the Swedish Transport Agency (Transportstyrelsen) and in-kind participation of LFV. It is also supported by the SESAR Joint Undertaking under the European Union's Horizon 2020 research and innovation programme under grant agreement No 783287". aircraft leaves its cruising level at the optimum top of descent and avoids level-off segments after that.

In this paper, we study correlation between the punctuality of arrivals for the major Swedish airport Arlanda in 2018, and the following weather phenomena: wind, visibility and CAPE within the terminal manoeuvring area (TMA). We calculate delay statistics and additional fuel burn due to deviations from the flight plans. Furthermore, we compute VFE within Arlanda TMA, together with the associated fuel waste.

The rest of the paper is organized as follows. In Section 2 we review related work on the topic and provide background information on the methods we use for analysis of the performance of Stockholm Arlanda airport arrivals.

We present the results of data analysis in Section 3 and summarize our findings in Section 4.

\section{Background}

This section reviews previous work and provides background information related to analysis of the weather impact on the flight efficiency of airport arrivals.

\subsection{Related Work}

Classification and analysis of causes of airport delays was a topic of interest for many years. In early works [3], [4] weather uncertainties are mentioned as the main contributor to the deviations in airport schedules. According to [1] airport ATFM delays $(19,704 \mathrm{~min} /$ daily) increased by $6.5 \%$ compared to May 2018 which had high delays due to weather and ATC industrial action.

Impact of deep convection and thunderstorms is also subject to ongoing research, e.g. Steiner et al. [29], [30] and Song et al. [27] investigated its implication both on the en-route flow management and for terminal area applications. Klein et al. [15] used a high-level airport model to quantify the impact of weather forecast uncertainty on delay costs.

Recent works [22], [31] confirmed the relevance and emphasized the importance of quantification and analysis of the weather impact on airport operation.

EUROCONTROL developed the methodology used by its Performance Review Unit (PRU) for the analysis of VFE during climb and descent [12]. Performance Review 
Commission of EUROCONTROL made an assessment of air traffic management in Europe for the year 2018, where among other indicators reviewed air traffic punctuality and vertical flight inefficiency at the top 30 European airports, including Stockholm airport Arlanda [10]. In addition, EUROCONTOL PRU continues working on the development and maintenance of the open access cloud based data repositories to enable stakeholders to reproduce the performance review results [28]. EUROCONTROL Experimental Center also develops new performance indicators targeting to capture different aspects of flight inefficiencies in TMA [6], [18], some of which we use in this work.

In [23] fuel consumption is evaluated for terminal areas with a Terminal Inefficiency metric based on the variation in terminal area fuel consumed across flights, reported by a major U.S. airline. Using this metric they quantify the additional fuel burn caused by Air Traffic Management (ATM) delay and terminal inefficiencies.

Estimation of the flight inefficiencies in terms of extra fuel burn calculated based on the algorithm proposed in [5] was considered in the scope of APACHE project (a SESAR 2020 exploratory research project) [20], [19], but mostly for en-route flight phase. Later Prats et al. [21] proposed a family of performance indicators to measure fuel inefficiencies. In this work, we apply similar techniques to fuel estimation during the descent phase within TMA.

Furthermore, in [13] and [32], an analysis of fuel savings of the Continuous Descent Operations (CDO) with respect to conventional procedures is analyzed. A reduction in fuel consumption of around $25-40 \%$ by flying CDO was reported.

\subsection{Weather Phenomena and Metrics}

We consider three weather metrics: wind gust, visibility and CAPE.

2.2.1. Wind Gust, Visibility. We use surface level measurements of wind gust and visibility, expressed in meters per second and meters respectively. Depending on cloud ceiling and runway visual range the spacing of aircraft on final approach must be increased. Low visibility reduces the runway capacity for landing aircraft. If this happens during a traffic peak hour, it causes major disruptions.

2.2.2. Convective Available Potential Energy (CAPE). CAPE is the energy a parcel of air has for upward motion, measured in joules per kilogram of air $(\mathrm{J} / \mathrm{kg})$. The higher the CAPE, the faster and higher the air parcel can rise. Most thunderstorms form in moderately unstable conditions (CAPE up to $1000 \mathrm{~J} / \mathrm{kg}$ ) but any value greater than $0 \mathrm{~J} / \mathrm{kg}$ indicates instability and an increasing possibility of thunderstorms and severe straight line winds.

\subsection{KPIs}

Here we list all the performance indicators we used in this work to evaluate TMA performance and explain how they are calculated.
2.3.1. Average Arrival Delay. First, we compute the average over the arrival delays for all flights delayed during the day of consideration. The delay is calculated as a difference between the scheduled arrival time and the actual time of arrival of the given flight.

2.3.2. ICAO KPIs. We use two KPIs proposed by the International Civil Aviation Organization (ICAO) [14]: Arrival punctuality and Level-off during descent.

According to ICAO, arrival punctuality ( $K P I 14.2 b)$ is calculated as the percent of the flights arriving at the gate on-time (delayed less than 15 minutes according to the schedule). We use the inverse version of this KPI, i.e. percent for flights delayed more than 15 minutes.

Vertical inefficiencies during the descent phase result from the inability of flights to keep up CDO (Continuous Descent Operations). This type of operations enables the execution of a flight profile optimized to the operating capability of the aircraft, giving as a result optimal continuous engine-idle descents (without using speedbreaks) that reduce fuel consumption, gaseous emissions and noise nuisance. If the aircraft levels at intermediate altitudes before landing, this descent is considered as vertical inefficient.

For evaluation of VFE we consider KPI19.2, the average time flown in level flight inside TMA using the techniques proposed by EUROCONTROL in [12] with small changes. We identify the point of the trajectory in which the aircraft enters the TMA and use it as a starting point for the calculations (instead of the Top of Descent (ToD), which may lie outside of TMA). A level segment is detected when the aircraft is flying with the vertical speed below the certain threshold. We use the value of 300 feet per minute for this threshold, the minimum time duration of the level flight is considered 30 seconds, and these 30 seconds are subtracted from each level duration as suggested in [12].

2.3.3. Additional Time in TMA. The additional time is calculated as the difference between the actual transit time and the time according to the flight plan. As stated in [18], it represents the extra time generated by the arrival management and "is a proxy for the level of inefficiency (holding, sequencing) of the inbound traffic flow during times when the airport is congested."

2.3.4. Fuel-Based PIs. Fuel-based PIs capture inefficiencies on tactical ATM layer in vertical domain as explained in [21]. The objective is to compare the fuel consumption of CDO trajectories with the actual flown trajectories. Fuel-based performance indicators are calculated using the 4.2 version of the Base of a Aircraft Data (BADA) [11].

The first expression used, known as the Total-Energy Model, equates the rate of work done by forces acting on the aircraft to the rate of increase in potential and kinetic energy, that is:

$$
(T-D) V_{T A S}=m g \frac{d h}{d t}+m V_{T A S} \frac{d V_{T A S}}{d t}
$$

Here $T$ is the thrust acting parallel to the aircraft velocity vector, $D$ is the aerodynamic drag, $m$ is the aircraft 
mass, $h$ is the geodetic altitude, $g$ is the gravitational acceleration and $V_{T A S}$ is the true airspeed.

The drag force is computed as follows:

$$
D=\frac{1}{2} \cdot \delta \cdot p_{0} \cdot \kappa \cdot S \cdot M^{2} \cdot C_{D}
$$

Here $\delta$ is the pressure ratio, $p_{0}$ is the standard atmospheric pressure at mean sea level (MSL), $\kappa$ is the adiabatic index of air, $S$ is the wing reference area, $M$ is the Mach number and $C_{D}$ is the drag coefficient. BADA proposes equations for computing $C_{D}$ depending on the aircraft configuration, and modelled as a polynomial of lift coefficient $C_{L}$.

Three separate thrust models are proposed in BADA, depending on the engine type: turbofan, turboprop or piston. Each model includes the contribution from all engines and provides the thrust as a function of airspeed, throttle setting and atmospheric conditions. The general formula of the thrust force, $T$, is:

$$
T=\delta \cdot W_{\text {mref }} \cdot C_{T}
$$

Here $\delta$ is the pressure ratio, $m_{r e f}$ is the reference mass (obtained from the Propulsive Forces Model (PFM)), $W_{\text {mref }}$ is the weight force at $m_{\text {ref }}$ and $C_{T}$ is the thrust coefficient, which is a function of Mach number.

For the three engine types, BADA proposes different equations to compute the thrust coefficient $C_{T}$ depending on the engine rating: maximum climb, maximum cruise, idle and no rating (direct throttle parameter input).

For estimation of the fuel consumption, BADA proposes once again a different model depending on the engine type, and also depending on the engine rating. Each model includes the contribution from all engines and provides the fuel consumption as a function of airspeed, throttle parameter and atmospheric conditions. The general formula for the fuel consumption, $F$, is:

$$
F=\delta \cdot \theta^{\frac{1}{2}} \cdot W_{m r e f} \cdot a_{0} \cdot L_{H V}^{-1} \cdot C_{F}
$$

Here $\delta$ is the pressure ratio, $\theta$ is the temperature ratio, $a_{0}$ is the speed of sound at MSL in standard atmosphere, $L_{H V}$ is the fuel lower heating value (obtained from the PFM) and $C_{F}$ is the fuel coefficient, which depends on thrust for non-idle ratings. For each aircraft model, BADA provides an xml file with the corresponding aircraft performance data. For instance, the coefficients used to compute the thrust coefficient $C_{T}$ of the thrust equation (3) are in this file. With the equations stated above, and the $\mathrm{xml}$ files for each aircraft, it is possible to compute the fuel consumption of a trajectory. The process followed is detailed below:

- Thrust computation: if the aircraft is climbing, max climb rating is chosen and the corresponding thrust formula (depending on the engine type) is applied. If the aircraft is descending, an idle rating is assumed. In level-offs, the total-energy model (equation (1)) is used in order to compute the corresponding aircraft thrust (drag is computed previously with equation (2)).

- Fuel consumption computation: for non-idle ratings, the thrust computed in the previous step is used to obtain the fuel coefficient $C_{F}$ used in equation (4). For descents, idle rating is assumed.

Wind was considered when computing the fuel consumption, and it was obtained from historical weather data (detailed in section 3.1). Furthermore, a $90 \%$ of the maximum landing mass has been assumed at the destination airport for all aircraft.

Generation of CDO trajectories. In order to generate the CDO trajectories an optimal control problem has to be solved as explained in details in [25]. First, a state vector with the initial conditions is needed. In this paper, it has been chosen as $\boldsymbol{x}=[v, h, s]$, where $v$ is the true airspeed, $h$ - the altitude of the aircraft, and $s$ - the distance to go. In order to obtain environmentally friendly trajectories, idle thrust is assumed and speed-brakes use is not allowed throughout the descent. In such conditions, the flight path angle is the only control variable in this problem $(\boldsymbol{u}=[\gamma])$, which is used to manage the energy of the aircraft and achieve different times of arrival at the metering fix with minimum fuel consumption and noise nuisance.

The dynamics of $\boldsymbol{x}$ are expressed by the following set of ordinary differential equations, considering a point-mass representation of the aircraft reduced to a "gamma-command" model, where vertical equilibrium is assumed (lift balances weight). In addition, the cross and vertical components of the wind are neglected, and the aerodynamic flight path angle is assumed to be small (i.e., $\sin \gamma \simeq \gamma$ and $\cos \gamma \simeq 1$ ):

$$
\boldsymbol{f}=\left[\begin{array}{c}
\dot{v} \\
\dot{h} \\
\dot{s}
\end{array}\right]=\left[\begin{array}{c}
\frac{T_{i d l e}-D}{m}-g \gamma \\
v \gamma \\
v+w
\end{array}\right]
$$

where $T_{\text {idle }}: \mathbb{R}^{n_{x}} \rightarrow \mathbb{R}$ is the idle thrust; $D: \mathbb{R}^{n_{x} \times n_{u}} \rightarrow$ $\mathbb{R}$ is the aerodynamic drag; $g$ is the gravity acceleration; $w$ is the wind and $m$ - the mass, which is assumed to be constant because the fuel consumption during an idle descent is a small fraction of the total $m$ [7]. The longitudinal component of the wind $w: \mathbb{R} \rightarrow \mathbb{R}$ is modelled by a smoothing spline [8]:

$$
w(h)=\sum_{i=1}^{n_{c}} c_{i} B_{i}(h)
$$

$B_{i}, i=1, \ldots, n_{c}$, are the $\mathrm{B}$-spline basis functions and $\boldsymbol{c}=\left[c_{1}, \ldots, c_{n_{c}}\right]$ are control points of the smoothing spline. It should be noted that the longitudinal wind has been modelled as a function of the altitude only, as done in similar works [9]. The control points of the spline approximating the longitudinal wind profile are obtained by fitting historical weather data (detailed in section 3.1).

In this paper, the trajectory is divided in two phases: the latter part of the cruise phase prior the ToD, and the idle descent down to the metering fix. Assuming that the original cruise speed will not be modified after the optimization process, the two-phases optimal control problem can be converted into a single-phase optimal control problem as follows:

$$
J=\frac{f}{v_{\text {cruise }}}+\int_{t_{0}}^{t_{f}}\left(f_{\text {idle }}+\mathrm{CI}\right) d t
$$


where $f: \mathbb{R}^{n_{x} \times n_{u}} \rightarrow \mathbb{R}$ and $f_{\text {idle }}: \mathbb{R}^{n_{x}} \rightarrow \mathbb{R}$ are the nominal and idle fuel flow, respectively; and CI is the cost index, which is a parameter chosen by the airspace user that reflects the relative importance of the cost of time with respect to fuel costs [2]. The CI is estimated by assuming that the aircraft was flying at the optimal speed in the cruise phase, as shown in [24].

To generate the optimum trajectories, five input parameters are used: aircraft model, cruise altitude, distance to go (i.e., the distance remaining to the metering fix by following a given route), speed (i.e., the true airspeed of the aircraft in cruise), and the cost index.

\section{Results}

This section describes the data used in this work and presents the results of data analysis for the Stockholm Arlanda airport in the year 2018.

\subsection{Data}

In this work we use multiple sources of historical data related to the performance of Stockholm Arlanda airport in 2018. Flight plans are obtained from the Demand Data Repository (DDR2, m1 file format) hosted by EUROCONTROL. For the historical flight trajectories we use DDR2 (m3 file format) and the Historical Database of the OpenSky Network [17], [26]. The historical weather data is provided by the National Oceanic and Atmospheric Administration (NOAA) through the National Operational Model Archive and Distribution System (NOMADS) [16].

Aircraft performance parameters for CDO trajectory generation are inputed from BADA 4.2 [11]. In the case the aircraft model does not correspond to any of the BADA models, a comparable aircraft in terms of performance and dimensions is used.

\subsection{Analysis of the Weather Impact on TMA Performance}

We compare the punctuality statistics and additional time in Stockholm Arlanda TMA based on DDR2 data with the weather statistics of wind speed, visibility and CAPE values for the same dates. To calculate VFE KPI (time flown level) we use OpenSky Network states data as it provides more accurate vertical profile.

First, we plot the data (metrics and KPIs) by days of the year. At some days we observe strong dependencies of the chosen KPIs from the considered weather metrics, while at other days the dependency is weak. This can be explained by the influence of some other operational factors or weather phenomena not considered in this study. For example, in February 2018 (Figure 1) we can see that the changes in wind gust enforce the increase of average additional time and average time flown level inside TMA. Low visibility events can increase the TMA KPIs, which is clearly the case on February 25. In July 2018 (Figure 2) high values of the punctuality-related KPIs (average delay and percent of delayed flights) coincide on some dates with increased gust, for example on July 27. From July
10 to the end of the month punctuality KPIs follow the changes of CAPE.

We continue with the deeper analysis of the dependencies between the KPI-metric pairs by introducing the thresholds in the weather metrics. Because of the nature of different weather events they can influence the flight performance differently. Strong wind gust makes aircraft landing problematic or simply impossible especially when it is above $12 \mathrm{~m} / \mathrm{s}$ (confirmed by the data analysis). This obviously results in higher values of all KPIs chosen in this study, which is shown in Figure 3.

We discovered, that with visibility lower than $5 \mathrm{~km}$ significantly more flights are delayed, which indicates that there is an indirect dependency between the corresponding KPI and visibility. Similarly, we observe direct dependencies between all the KPIs and the other two weather metrics.

To examine the relationship between KPIs and weather metrics we apply regression analysis to the data for the whole year 2018. We clean the data by removing the KPI outliers. To remove skewness in weather metrics distribution we filter out data with the boundary values (more than $24 \mathrm{~km}$ for visibility and less than $100 \mathrm{~J} / \mathrm{kg}$ for CAPE).

Simple linear regression demonstrates moderate dependency between average delay and CAPE (coefficient $=0.0045$, intercept $=6.1786$, $\left.R^{2}=0.5217, R_{a d j}^{2}=0.5052, R M S E=1.8189\right)$, which is illustrated in Figure 4. While the average delay and visibility show weak dependency in simple regression (coefficient $=-0.0134$, intercept $=8.3003, R^{2}=0.0013, R_{\text {adj }}^{2}=-0.0131$, $R M S E=2.3042$ ), joining these two metrics in multiple linear regression results in better fitted model (coefficients $=[-0.1422,0.0054]$, intercept $=6.8777$ $\left.R^{2}=0.7816, R_{a d j}^{2}=0.7524, R M S E=1.4956\right)$. Figures 5 and 6 illustrate the corresponding results.

\subsection{Additional fuel burn}

In order to assess fuel efficiency within Arlanda TMA during the year 2018, we calculate the fuel waste associated with the vertical flight inefficiency for individual descent profiles within TMA. For actual trajectories we use both DDR2 and Opensky Network tracks and compare the results for additional fuel burn. The objective is to compare the fuel consumption of CDO trajectories with the actual flown trajectories. The CDO were only optimized for the vertical plane, so the distance to go was obtained from either DDR or Opensky.

First, we compare the fuel consumption of the actual trajectories obtained from DDR2 $\mathrm{m} 3$ data with the CDO profiles obtained with the trajectory optimization technique explained in 2.3.4. We calculate the additional fuel burn per day for all Arlanda airport arrivals during the months of February and July 2018, where we have discovered dependencies between the weather events and several KPIs. Figures 7 and 8 illustrate the results. We calculate that in both months CDO provide a reduction of fuel consumption around 60-65\%, which constitutes significant inefficiency of the vertical profiles actually flown during these months. 

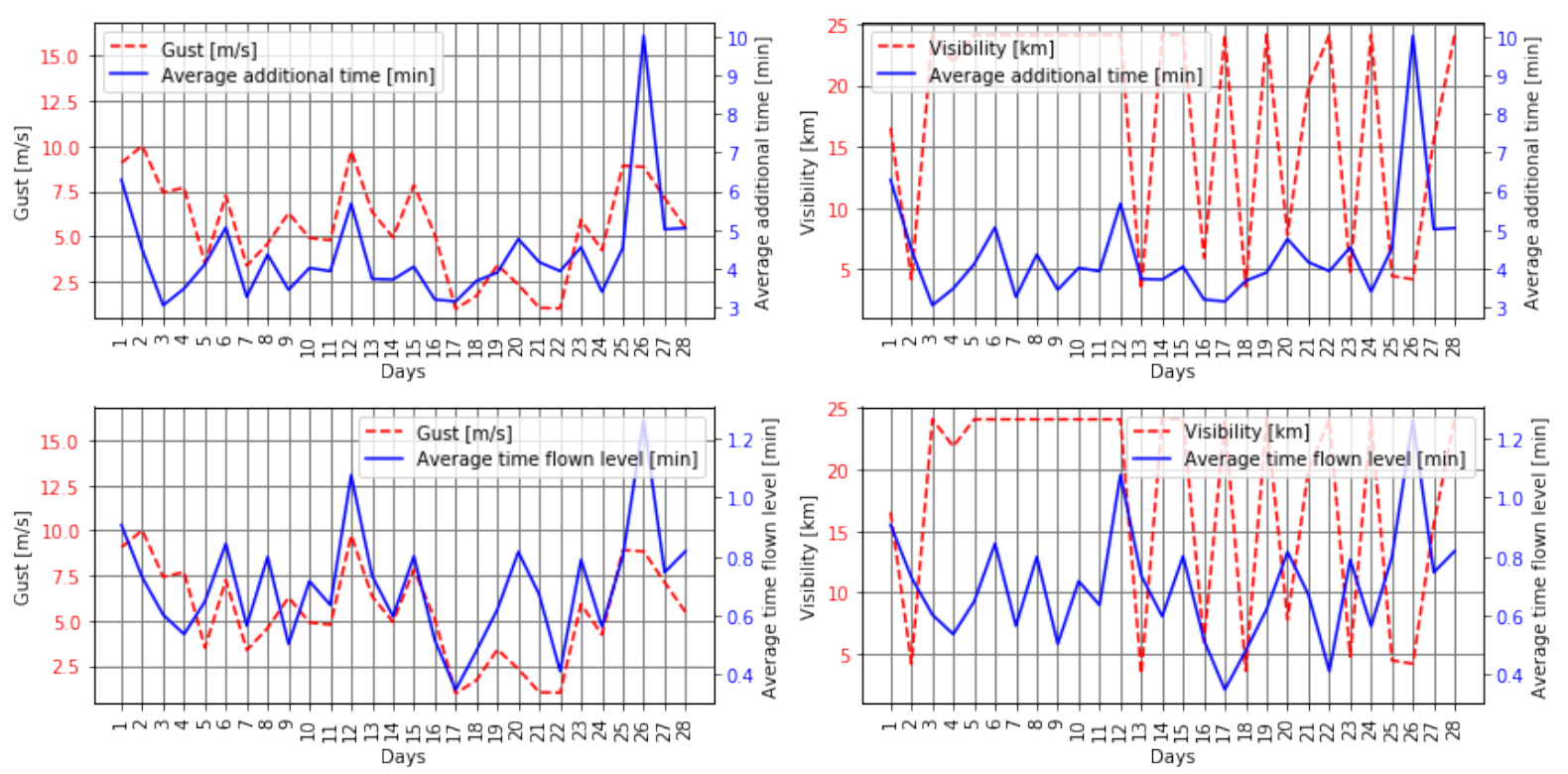

Figure 1. Weather metrics (wind gust and visibility) and KPIs (average additional time in TMA and average time flown level) for the month of February 2018.
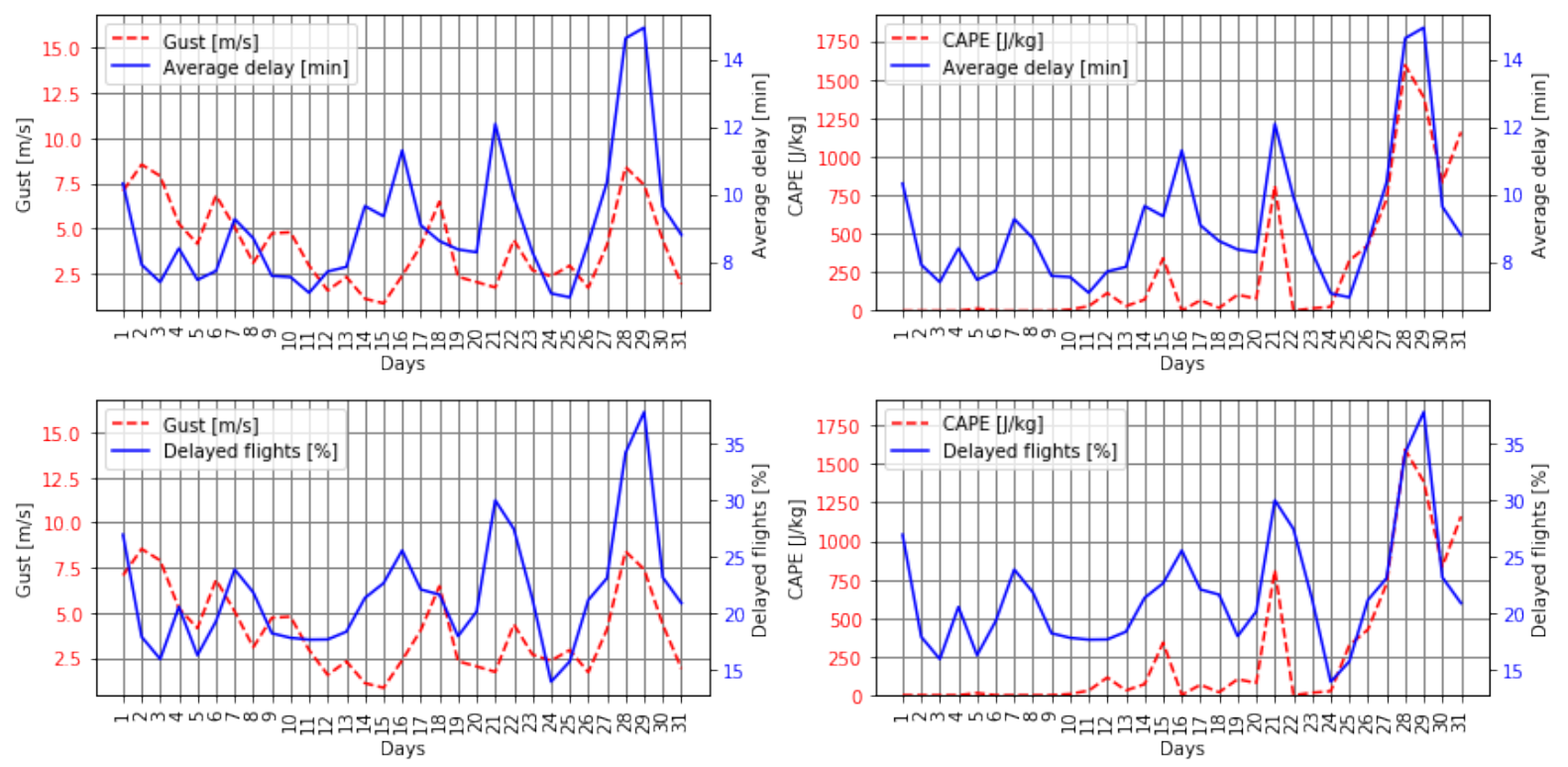

Figure 2. Weather metrics (wind gust and CAPE) and KPIs (average delay and percent of delayed flights) for the month of July 2018.

It is important to recall that we calculate the fuel inside TMA only; if the whole descent was compared, the difference would have been lower, as level-offs at lower altitudes are more detrimental for efficiency than those at higher altitudes.

Similar computations have been made by using Opensky data for the month of February and July 2018 (Figures 9 and 10). While the fuel consumption is higher than with DDR (usually the difference is between $1 \%$ and $10 \%$ ), the additional fuel burn with respect to $\mathrm{CDO}$ remains almost the same.

Absolute values for the fuel consumption are shown in Figures 11 and 12, representing the average fuel consumption over the flights per day during the months of February and July 2018.
Fuel consumption suffers significant changes throughout the month. First of all, the calculation took into account wind components, which demonstrated the impact on the efficiency of vertical profiles. Hence, we observe the decrease of the fuel consumption during the corresponding days with the lowest gust values reported (e.g. on February 17, Figure 1, gust-average time flown level plot), and increase of the fuel consumption during the days with the highest gust values (e.g. February 2526). Moreover, the increase of the fuel burn during the days with low visibility (e.g. the same days February 2526, visibility-time flown level plot on Figure 1) can be a result of Low Visibility Procedures and the connected changes in the descent profiles because of the difficult weather condition. 

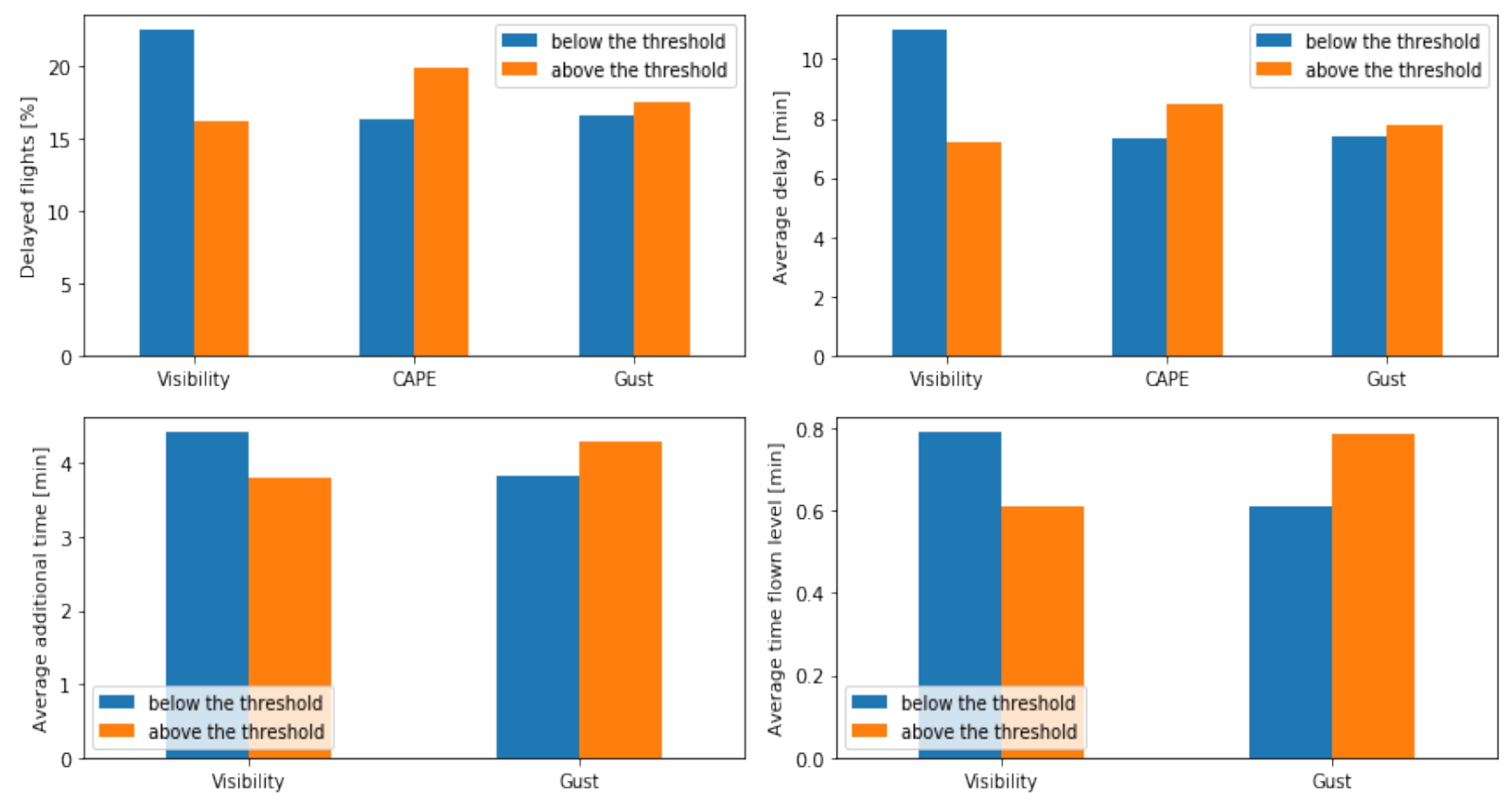

Figure 3. Average KPIs over the year 2018 calculated with respect to the following thresholds in the weather metrics: visibility - 5 km, CAPE $100 \mathrm{~J} / \mathrm{kg}$ and gust $-12 \mathrm{~m} / \mathrm{s}$.

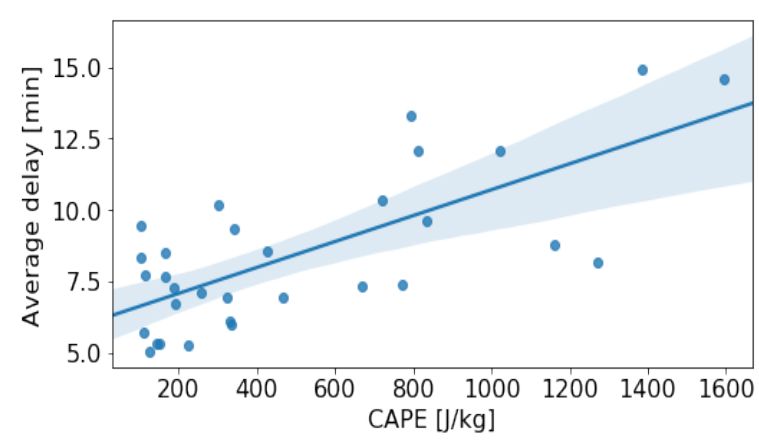

Figure 4. Simple linear regression for average delay versus CAPE.

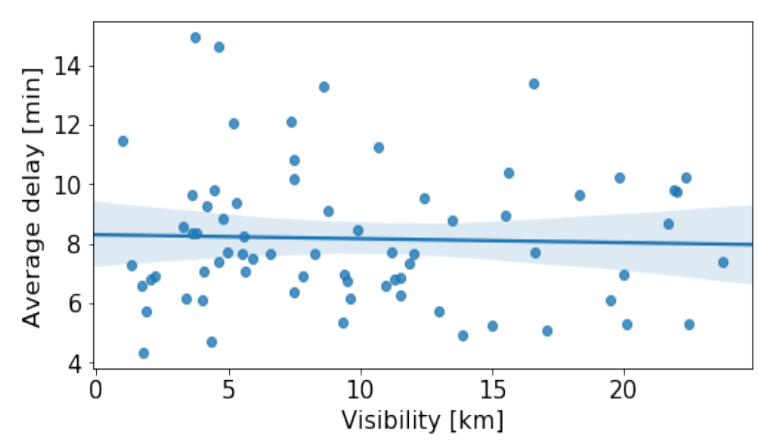

Figure 5. Simple linear regression for average delay versus visibility.

The extreme CAPE values on 28-29 of July (CAPE curves on plot Figure 2) result in the increased time flown level (the figure is not presented here), and correspondingly increases the fuel burn at the same days that can be explained by changed landing procedures during the days with high convective instabilities.

Finally, it is important to highlight the advantages

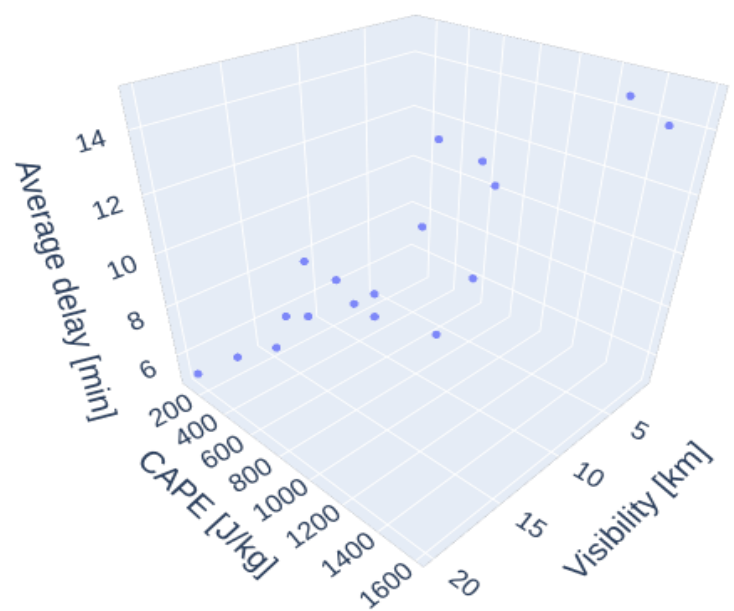

Figure 6. Multiple linear regression for average delay versus visibility and CAPE.

(and some disadvantages) that Opensky data represents over DDR data. First, the better data granularity of Opensky data makes it a better option to estimate fuel consumption inside TMA. While DDR usually provides only 3 or 4 segments inside TMA, in Opensky there are about 60-80 waypoints (depending on the trajectory), which makes it more reliable. However, there are also some errors in Opensky data, which tend to cause very high values when computing the fuel consumption. Several data outliers were found in Opensky tracks. For instance, in some trajectories there are some repeated waypoints, even with the time advancing (which would mean the aircraft remains still, which is not possible). There are other situations where the latitude and longitude do not seem to correspond to the trajectory we are dealing with, and some of the speed values that could be extracted from 


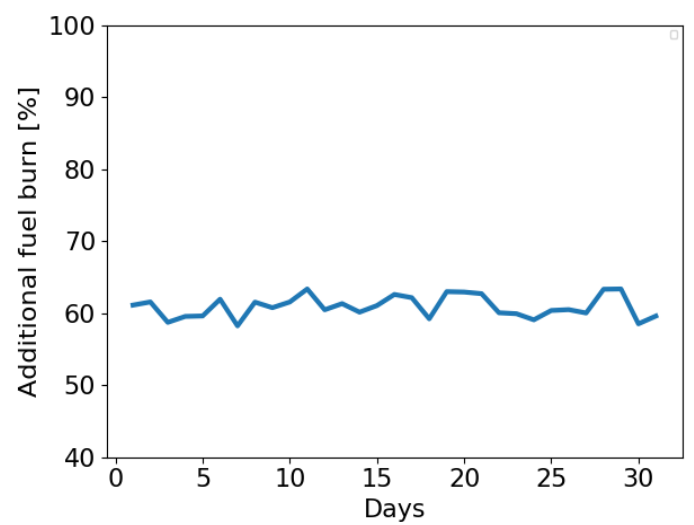

Figure 7. Additional fuel burn (in percent per day) due to inefficient vertical profiles, calculated as the difference between the actual flown trajectories (DDR m3) and the optimal trajectories, in total fuel consumption per day inside TMA for the month of February 2018.

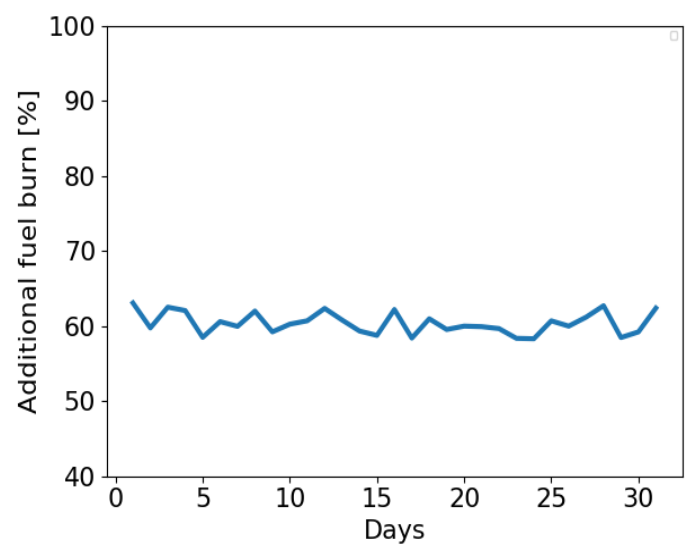

Figure 8. Additional fuel burn (in percent per day) due to inefficient vertical profiles, calculated as the difference between the actual flown trajectories (DDR m3) and the optimal trajectories, in total fuel consumption per day inside TMA for the month of July 2018.

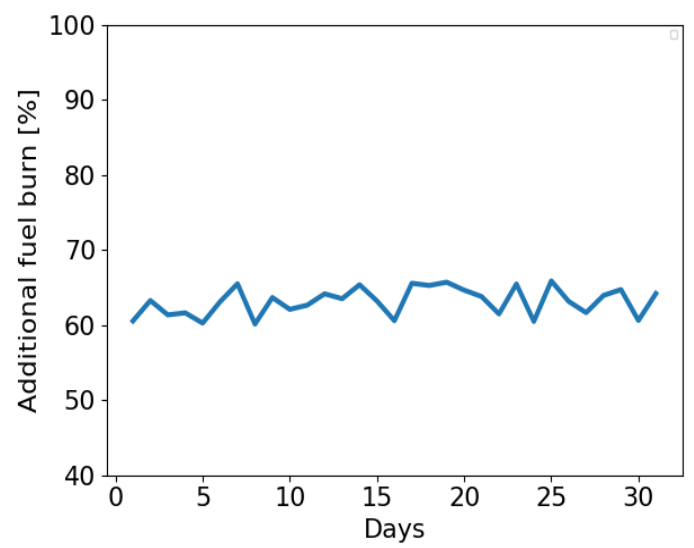

Figure 9. Additional fuel burn (in percent per day) inside TMA due to inefficient vertical profiles, calculated as the difference between the actual flown trajectories (Opensky tracks) and the optimal trajectories, for the month of February 2018.

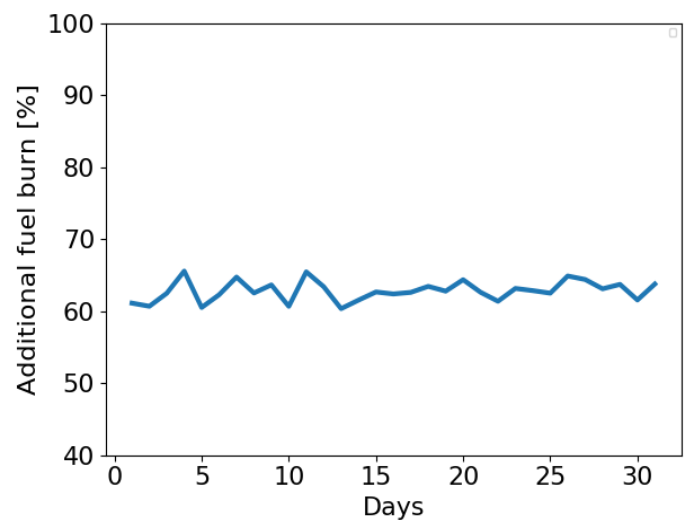

Figure 10. Additional fuel burn (in percent per day) inside TMA due to inefficient vertical profiles, calculated as the difference between the actual flown trajectories (Opensky tracks) and the optimal trajectories, for the month of July 2018.

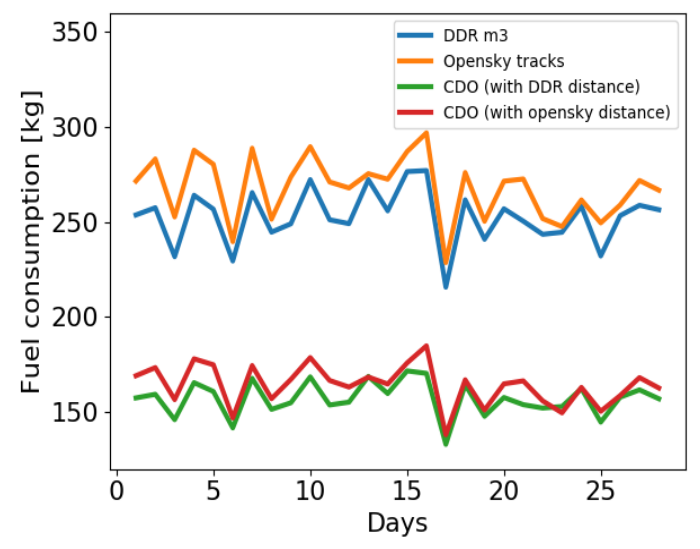

Figure 11. Average fuel consumption over the flights per day (in $\mathrm{kg}$ ) for actual flown trajectories (DDR m3 and Opensky tracks) and CDO within TMA for arrival flights in Stockholm Arlanda during the month of February 2018.

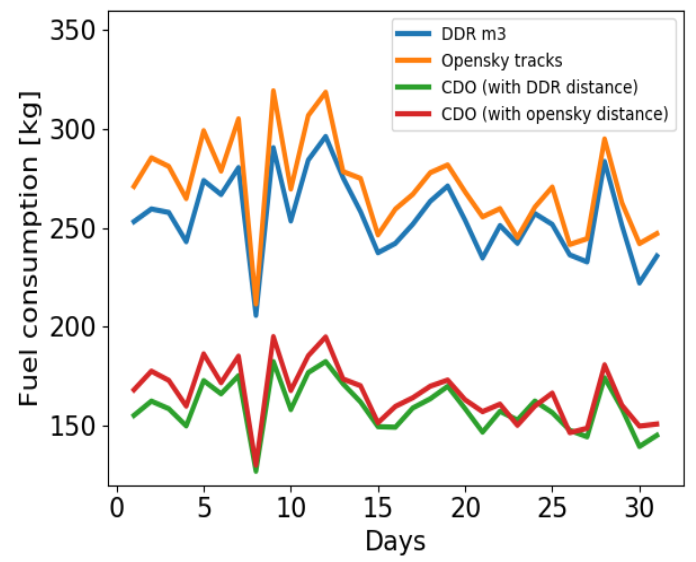

Figure 12. Average fuel consumption over the flights per day (in $\mathrm{kg}$ )for actual flown trajectories (DDR m3 and Opensky tracks) and CDO within TMA for arrival flights in Stockholm Arlanda during the month of July 2018. 
Opensky tracks are wrong too. Since all these problems were found in less than $1 \%$ of flights, in this work we chose to remove these flights in order to have proper fuel consumption values. However, in future work it would be interesting to find an outlier removal method in order to efficiently solve this problem.

\section{Conclusions}

In this paper we studied how different weather phenomena influence arrival punctuality and VFE on example of Stockholm Arlanda airport. We analyzed the dependencies between certain KPI-weather metric pairs. Performance degradation during the days when such weather events as low visibility, strong gusts or high thunderstorm probability were detected, result in significant amount of additional fuel burn. Our calculations show that CDO provide a reduction of fuel consumption around $60-65 \%$, which constitutes significant inefficiency of the vertical profiles actually flown during the observed months.

In addition, we shared our experience working with DDR and OpenSky data sources and discussed their applicability for calculations inside TMA, outlining the advantages and disadvantages of both.

The results of this work create a base for future studies of the impact of different factors such as ATM automation or different weather conditions (snow, icing) on the arrival delays, vertical efficiency and associated fuel waste.

\section{Copyright Statement}

The authors confirm that they, and/or their company or institution, hold copyright of all original material included in their paper. They also confirm they have obtained permission, from the copyright holder of any third party material included in their paper, to publish it as part of their paper. The authors grant full permission for the publication and distribution of their paper as part of the EIWAC2019 proceedings or as individual off-prints from the proceedings.

\section{References}

[1] European Commision Network Manager. Monthly Network Operations Report, May 2019.

[2] Airbus. Getting to grips with the cost index - Issue II. Technical Report 2, 1998.

[3] S. Allan, J. Beesley, J. Evans, and S. Gaddy. Analysis of delay causality at newark international airport. In ATM Seminar, 2001.

[4] R. Beatty, R. Hsu, L. Berry, and J. Rome. Preliminary evaluation of flight delay propagation through an airline schedule. Air Traffic Control Quarterly, 7(4):259-270, 1999.

[5] G. B. Chatterji. Fuel burn estimation using real track data. In 11th AIAA Aviation Technology, Integration, and Operations (ATIO) Conference, including the AIAA Balloon Systems Conference and 19th AIAA Lighter-Than, page 6881, 2011.

[6] R. Christien, E. Hoffman, and K. Zeghal. Spacing and pressure to characterise arrival sequencing. In ATM Seminar, 2019.

[7] J. P. Clarke, N. T. Ho, L. Ren, J. Brown, K. Elmer, K. F. Zou, C. Hunting, D. McGregor, B. Shivashankara, K. Tong, A. W. Warren, and J. Wat. Continuous descent approach: Design and flight test for Louisville international airport. J. of Aircraft, 41(5):1054-1066, 2004.
[8] C. de Boor. On calculating with B-splines. Journal of Approximation Theory, 6(1):50-62, 1972.

[9] P. M. A. de Jong, J. J. van der Laan, A. C. Veld, M. M. van Paassen, and M. Mulder. Wind-Profile Estimation Using Airborne Sensors. Journal of Aircraft, 51(6):1852-1863, 2014.

[10] EUROCONTROL. Performance Review Report: An Assessment of Air Traffic Management in Europe during the Calendar Year 2018 (Draft Final Report for consultation with stakeholders).

[11] EUROCONTROL. User Manual for the Base of Aricraft Data (BADA) Family 4, 2014.

[12] EUROCONTROL. Analysis of vertical flight efficiency during climb and descent, 2017.

[13] H. Fricke, C. Seiss, and R. Herrmann. Fuel and energy benchmark analysis of continuous descent operations. In ATM Seminar, 2015.

[14] KPI Overview. https://www4.icao.int/ganpportal/ASBU/KPI, last accessed 29.08.2019.

[15] A. Klein, S. Kavoussi, and R. S. Lee. Weather forecast accuracy: Study of impact on airport capacity and estimation of avoidable costs. In ATM Seminar, 2009.

[16] NOAA. https://www.ncdc.noaa.gov/data-access/modeldata/model-datasets/global-forcast-system-gfs, last accessed 29.08.2019.

[17] OpenSky Network. https://opensky-network.org, last accessed 29.08.2019.

[18] P. Pasutto, E. Hoffman, and K. Zeghal. Vertical efficiency in descent compared to best local practices. In ATM Seminar, 2019.

[19] X. Prats, I. Agüi, F. Netjasov, G. Pavlovic, and A. Vidosavljevic. APACHE-Final project results report. 2018.

[20] X. Prats, C. Barrado, F. Netjasov, D. Crnogorac, G. Pavlovic, I. Agüi, and A. Vidosavljevic. Enhanced indicators to monitor atm performance in europe. In SIDs, 2018.

[21] X. Prats, R. Dalmau, and C. Barrado. Identifying the sources of flight inefficiency from historical aircraft trajectories. In ATM Seminar, 2019.

[22] S. Reitmann, S. Alam, and M. Schultz. Advanced quantification of weather impact on air traffic management. In ATM Seminar, 2019.

[23] M. S. Ryerson, M. Hansen, and J. Bonn. Time to burn: Flight delay, terminal efficiency, and fuel consumption in the national airspace system. Transportation Research Part A: Policy and Practice, 69:286-298, 2014.

[24] R. Sáez, R. Dalmau, and X. Prats. Optimal assignment of 4D close-loop instructions to enable CDOs in dense TMAs. In DASC, 2018.

[25] R. Sáez, X. Prats, T. Polishchuk, V. Polishchuk, and C. Schmidt. Automation for Separation with CDOs: Dynamic Aircraft Arrival Routes. In ATM Seminar, 2019.

[26] M. Schäfer, M. Strohmeier, V. Lenders, I. Martinovic, and M. Wilhelm. Bringing Up OpenSky: A Large-scale ADS-B Sensor Network for Research. In IPSN'14, 2014.

[27] L. Song, D. Greenbaum, and C. Wanke. The impact of severe weather on sector capacity. In ATM Seminar, 2009.

[28] E. Spinielli, R. Koelle, K. Barker, and N. Korbey. Open Flight Trajectories for Reproducible ANS Performance Review. In SIDs, 2018.

[29] M. Steiner, R. Bateman, D. Megenhardt, Y. Liu, M. Xu, M. Pocernich, and J. Krozel. Translation of ensemble weather forecasts into probabilistic air traffic capacity impact. Air Traffic Control Quarterly, 18(3):229-254, 2010.

[30] M. Steiner, W. Deierling, K. Ikeda, E. Nelson, and R. Bass. Airline and airport operations under lightning threats-safety risks, impacts, uncertainties, and how to deal with them all. In 6th AIAA Atmospheric and Space Environments Conference, page 2900, 2014.

[31] M. Steinheimer, C. Kern, and M. Kerschbaum. Quantification of weather impact on air arrival management. In ATM Seminar, 2019.

[32] F. Wubben and J. Busink. Environmental Benefits of continuous descent approaches at Schiphol airport compared with conventional approach procedures. Technical report, National Aerospace Laboratory (NLR), 2000. 